\title{
Regionalisation of Major Trauma Services in England: A Post- Implementation Analysis
}

\section{Background}

There is strong evidence that severely injured patients are optimally treated within inclusive trauma networks. One feature of such networks is pre-hospital triage of the most injured patients to designated trauma hospitals. In April 2012, major trauma services across England were reconfigured into regional trauma networks based around designated "Major Trauma Centres" (MTCs). This study explored the early effects of major trauma service regionalisation in England.

\section{Methods}

The Trauma \& Audit Research Network (TARN) identified all severely injured patients treated in the nine months before and after each MTC became fully operational. Outcomes included quality indicators (e.g. time to CT scanning for head injured patients) and clinical outcomes (e.g. in-hospital mortality). Completeness of the TARN dataset was quantified using Hospital Episode Statistics (HES). Death registrations were used to identify any change in the overall number of traumatic deaths in England.

\section{Results}

The number of severely injured patients treated in MTCs increased from 7,705 to 12,476 . All care quality indicators improved: treatment by a consultant $(30.4 \%$ versus $54.3 \%, \mathrm{p}<0.001$ ), administration of tranexamic acid to bleeding patients (17.0\% versus $58.5 \%, \mathrm{p}=0.006)$, and time to CT scanning for head injured 
patients (49.2 versus 31.2 minutes, $\mathrm{p}<0.001$ ). Fewer patients required secondary transfer between hospitals (31.3\% versus $25.9 \%, \mathrm{p}<0.001)$ and a greater proportion were discharged with a GOS of "good recovery" (52.4\% versus 64.5\%, $\mathrm{p}<0.001)$. There were no differences in either crude or adjusted mortality between the periods for all patients or for those with an Injury Severity Score 215. The overall number of traumatic deaths in England did not change $(\mathrm{p}=0.566)$.

\section{Conclusions}

There is early evidence of improved care associated with trauma service regionalisation, including some process measures, enhanced case reporting for national audit, and reduced need for secondary transfer of patients between hospitals. However, in this early post-implementation analysis, significant reductions in patient mortality were not observed. 